\title{
INMIGRANTES, ESTRATEGIAS FAMILIARES $Y$ ARRAIGO: LAS LECCIONES DE LA CRISIS EN LAS ÁREAS RURALES.
}

\section{IMMIGRANTS, FAMILY STRATEGIES AND LONG-TERM SETTLEMENT: THE LESSONS OF THE CRISIS IN RURAL AREAS'.}

Rosario Sampedro Gallego* y Luis Camarero Rioja**

\begin{abstract}
Resumen: El asentamiento de población de origen extranjero en las áreas rurales españolas en las últimas décadas ha sido considerado como una oportunidad para la revitalización demográfica y social del mundo rural. La crisis económica que comienza en 2008 abre interrogantes sobre los factores que pueden favorecer o dificultar el arraigo rural. En este artículo analizamos la evolución de la población inmigrante en Castilla y León entre 2007 y 2014, utilizando datos de los Padrones Municipales y del Censo de Población de 2011. Relacionamos esta evolución con los modelos familiares de migración y de inserción laboral de cinco grupos distintos de inmigrantes según su origen nacional. Los datos sugieren que determinadas estrategias familiares - vinculadas a las familias transnacionales o al matrimonio con españoles - y determinados modelos de inserción laboral —con una clara especialización sectorial por género- se relacionan con la permanencia de los inmigrantes en las áreas rurales.
\end{abstract}

Departamento de Sociología y Trabajo Social. Universidad de Valladolid. mariarosario.sampedro@uva.es

** Departamento de Teoría, Metodología y Cambio Social. UNED. lcamarero@poli.uned.es

${ }^{1}$ Artículo realizado en el marco del proyecto "Crisis e inmigración en el medio rural de Castilla y León: Escenarios socio-territoriales para el arraigo de los inmigrantes y la sostenibilidad social de las áreas rurales" (CSO2015-67525-R), del Plan Nacional de I+D+i del Ministerio de Economía y Competitividad . 
Palabras clave: inmigración, medio rural, estrategias familiares, empleo, Castilla y León.

Abstract: The settlement of foreign born immigrants in Spanish rural areas in the last decades has been considered like an opportunity to stop depopulation and improve social and economic life in rural towns and villages. After the burst out of economic crisis in 2008 a lot of questions arise about factors that enhance or hinder the permanence of these newcomers in rural areas. In this paper we analyze the evolution of foreign born population in Castilla y Leon from 2007 to 2014, using data from Spanish Register of Inhabitants and from the Spanish Population Census of 2011. We relate the observed evolution of five national groups of immigrants with their family migration strategies and labour patterns. Data suggest that certain family migration strategies - those linked to transnational families and to marriage with Spaniards-and certain labour patterns - with a clear gendered pattern of employment-are connected with the permanence of immigrants in rural areas.

Keywords: immigration, rural areas, family strategies, employment, Castilla y León.

\section{INTRODUCCIÓN: MEDIO RURAL, INMIGRACIÓN Y CRISIS ECONÓMICA}

Durante la última década del pasado siglo y la primera del actual, nuestro país asistió a la llegada de un gran contingente de población inmigrante en edad activa que contribuyó a rejuvenecer la estructura demográfica, a reactivar la economía y a dar mayor solidez a un sistema de bienestar social que pivota en torno a la solidaridad intergeneracional (Arango, 1999). Aunque son los núcleos urbanos y las grandes áreas metropolitanas las que concentran la mayor proporción de población inmigrante, estos flujos afectan también de manera significativa a las áreas rurales, merced a la elevada implicación de los inmigrantes en sectores como la agricultura, la construcción o los servicios de proximidad (Pedreño, 1999; Hoggart y Mendoza, 1999; Mendoza, 2003; Muñoz, 2005).

Para muchas áreas rurales de nuestro país, especialmente en el interior peninsular, la llegada de población inmigrante ha supuesto una oportunidad para detener e incluso revertir un proceso de despoblamiento que duraba décadas (Esparcia, 2002; García Coll y Sánchez, 2005; Solé, 2010; Prieto y Papadodima, 2010; Collantes et 
al., 2014). La paralización de estos flujos migratorios desde el inicio de la crisis económica en 2008 abre interrogantes sobre hasta qué punto los núcleos rurales son capaces de retener a medio y largo plazo a estos nuevos pobladores.

La presencia de residentes extranjeros en las áreas rurales españolas responde a diferentes procesos migratorios y diferentes tipos de movilidad con impactos sociales y territoriales muy diversos. Sin embargo la mayor parte de los flujos migratorios internacionales hacia las áreas rurales en las dos últimas décadas se basa en una migración de tipo laboral, procedente del norte de África, América Latina y los países del Este de Europa, especialmente vinculada a las áreas de agricultura intensiva, y al desarrollo del turismo y la construcción (Camarero, Sampedro y Oliva, 2011).

Esta nueva ola migratoria de tipo laboral, muy diferente a las anteriores migraciones de retiro o de retorno presentes ya en décadas anteriores, es común a todos los países del sur de Europa (Kasimis, 2008; Fonseca, 2008; Kasimis, Papadoulos y Pappas, 2010) y responde a un modelo migratorio que se diferencia de modelos anteriores por la importancia que adquiere el empleo en sectores como la agricultura, la construcción y los servicios, la diversidad geográfica y cultural de los migrantes, el nivel considerable de empleo informal, y la coexistencia de los flujos migratorios con tasas significativas de desempleo en la población autóctona (King, 2000; Jentsch, 2009).

La distribución geográfica de los residentes extranjeros en España es bastante similar para áreas rurales y urbanas: son las regiones y provincias de la costa mediterránea oriental y sudoriental, las comunidades insulares, y las grandes áreas metropolitanas de Madrid y Barcelona y sus territorios adyacentes los que presentan una mayor y más temprana presencia de inmigración extranjera (Pinilla, Ayuda y Sáez, 2008). Pero a medida que avanza el nuevo siglo la presencia de inmigrantes extranjeros va haciéndose cada vez mayor en las ciudades y pueblos de las comunidades autónomas del interior y el norte peninsular, en una especie de, al menos aparente, efecto de desbordamiento desde las áreas más dinámicas (Roquer y Blay, 2008; Reher y Silvestre, 2011; Collantes et al., 2014). Es difícil saber si nos encontramos ante desplazamientos internos desde esas comunidades con una más precoz presencia migratoria hacia estos nuevos destinos, o si éstos comienzan a serlo ya para la población inmigrante en sus países de origen.

Si la inmigración extranjera ha sido considerada una oportunidad para detener la despoblación de las áreas rurales, esto es aún 
más evidente en aquellas regiones que experimentan un declive demográfico secular y tienen un importante componente territorial rural, como es el caso de Castilla y León. Aunque Castilla y León recibe de forma atenuada y con retraso la gran oleada de inmigración laboral de los años noventa, estos flujos demográficos han transformado significativamente unas dinámicas demográficas marcadas desde hace décadas por la despoblación y el envejecimiento (Consejo Económico y Social de Castilla y León 2012; Ibáñez, 2006).

Los estudios realizados hasta el momento coinciden en señalar la importante contribución de la inmigración extranjera a la reducción de la despoblación en los pequeños municipios. Sin embargo, se ha visto también cómo este proceso ha agudizado la dicotomía ya existente entre un medio rural dinámico y el a veces denominado "rural profundo". La población inmigrante tendería a concentrarse en municipios de tamaño medio, centros comarcales con unas economías más diversificadas, una mayor oferta de viviendas, buenas comunicaciones y unos mejores estándares de servicios públicos, contribuyendo a hacer más evidente el abandono de los núcleos más pequeños y aislados (Bayona y Gil, 2013; Consejo Económico y Social de Castilla y León, 2012)

Otro efecto relevante de la inmigración extranjera hacia las áreas rurales es la agudización de la masculinización rural (Camarero et al., 2009). El hecho de que los flujos migratorios sean en la mayor parte de los casos compuestos por más hombres que mujeres, especialmente los procedentes del norte de África, tiene esta repercusión en la estructura demográfica del medio rural.

Una cuestión clave en el análisis sociológico de las migraciones es la existencia de diferentes estrategias migratorias según el origen geográfico de los inmigrantes, estrategias que se expresan en la composición fundamentalmente femenina o masculina de los flujos migratorios, y en los procesos de reagrupación familiar, más o menos prolongados en el tiempo (Camarero y García, 2004; Camarero, 2010; Requena y Sánchez Domínguez, 2011; Sánchez Domínguez, de Valk y Reher, 2011; Sampedro, 2012). No sabemos demasiado acerca de cómo estas diferentes estrategias familiares están influyendo en el asentamiento de la población inmigrante en el medio rural, aunque algunos datos de la Encuesta Nacional de Inmigración de 2007 apuntaban a que la residencia rural está vinculada a formas de vida más familiares (Camarero, Sampedro y Oliva, 2013).

Por otro lado, el análisis de la movilidad residencial y ocupacional 
de los inmigrantes extranjeros sugiere que las áreas rurales y los trabajos agrícolas podrían ser solo una estación de paso en un camino hacia mejores empleos en entornos urbanos (Pumares, García y Asensio, 2006; Miguélez et al., 2011; Camarero, Sampedro y Oliva, 2013). Aunque es cierto que los datos confirman la existencia de un importante proceso de redistribución de la población inmigrante desde las capitales provinciales hacia otro tipo de municipios, especialmente los más pequeños (Reher y Silvestre, 2011), lo cierto es que el saldo resulta negativo para el medio rural: hay más probabilidad de que los inmigrantes residentes en primer lugar en núcleos rurales se trasladen a las capitales provinciales que a la inversa. Es decir, aunque los grandes centros urbanos se constituyen como puertas de entrada, desde las que se produce ese efecto de distribución territorial, el abandono de la agricultura y del medio rural se asociarían muy directamente con el deseo de movilidad y progreso social que acompaña a todo proyecto migratorio (Pedone, 2005, Pedreño y Riquelme, 2006).

Otros estudios apuntan sin embargo el importante papel que la población inmigrante tiene no solo en los trabajos agrarios de carácter altamente estacional e informal, sino en muchos otros empleos vinculados a la agroindustria o al comercio y los servicios de proximidad. La población inmigrante sostiene una actividad económica que la población autóctona no está en condiciones de asumir, y es protagonista de iniciativas empresariales que revitalizan la vida económica de los pueblos (Mancilla, Viladomiou y Guallarte, 2010; Sánchez Flores et al., 2014; Soronellas et al., 2014).

El interés sobre el efecto que a medio y largo plazo puede tener la inmigración extranjera en el medio rural ha crecido a medida que vamos conociendo los efectos de la crisis económica que comienza en 2008 y que supone un verdadero cambio de ciclo migratorio (Reher, Requena y Sanz, 2011). A nivel global se ha observado una reducción drástica de los flujos migratorios y una disminución del crecimiento de población de origen extranjero hasta tasas similares a las previas a la gran ola migratoria de los noventa. Pero esta reducción se debe más a la no llegada de nuevos efectivos que al retorno de la población residente, que se ha producido de forma paulatina y limitada. (Arango, 2012; del Moral y Martín, 2014).

En lo que respecta al medio rural, estudios recientes muestran que la crisis no ha propiciado una vuelta de la población - autóctona o inmigrante- al campo, sino que al contrario, los movimientos 
de población hacia los pequeños municipios han disminuido (Camarero, 2013). La sustitución de trabajadores inmigrantes por autóctonos en determinados sectores económicos del medio rural tampoco está teniendo las dimensiones esperadas (Ortiz, Arnalte y Moragues, 2013). Entre 2012 y 2014, sin embargo, los registros padronales ya dejan ver una reducción en números absolutos de la población de origen extranjero a nivel nacional y en todas las comunidades autónomas, lo que implica que el impacto de la crisis está teniendo ya unas consecuencias claras.

En definitiva, en este momento se abren múltiples interrogantes sobre el efecto que la crisis económica ha tenido en la presencia de población de origen extranjero en el medio rural, sobre la capacidad de diferentes tipos de núcleos y áreas rurales para retener a la población inmigrante, y sobre las condiciones sociales y económicas que favorecen más o menos la permanencia e integración de diferentes colectivos nacionales en unos territorios necesitados de esta población para asegurar su sostenibilidad social a medio plazo. El objetivo de este artículo es explorar estos interrogantes analizando la evolución de cinco grupos nacionales de inmigrantes de origen extranjero en Castilla y León, entre 2007 y 2014. En un primer momento se examinará la relación entre orígenes nacionales y pautas de asentamiento territorial; a continuación analizaremos las diferentes estrategias migratorias que se desprenden de la composición por sexo y las formas de convivencia familiar de dichos grupos; posteriormente analizaremos las pautas de empleo, para finalizar relacionando todas las dimensiones anteriores con la evolución de los cinco grupos nacionales en los años de la crisis.

\section{DATOS Y METODOLOGÍA}

Los cinco grupos nacionales considerados son los procedentes de Bulgaria, Rumanía, Marruecos, Colombia y Ecuador, los más numerosos en Castilla y León, una vez excluidos los extranjeros de la UE-15 (Portugal y Francia). Estos cinco grupos nacionales representan en el año 2007 el 44,7\% de toda la población nacida en el extranjero y residente en la comunidad autónoma, habiendo aumentado su peso proporcional en los años de la crisis hasta un 46,9\% en 2014. 


\section{TABLA 1}

NACIDOS EN EL EXTRANJERO EN CASTILLA Y LEÓN, POR PAÍS DE NACIMIENTO

(10 principales grupos nacionales en 2007)

\begin{tabular}{|l|r|c|c|c|}
\hline \multirow{2}{*}{ País de nacimiento } & \multicolumn{2}{|c|}{2007} & \multicolumn{2}{c|}{2014} \\
\cline { 2 - 5 } & Total & $\begin{array}{c}\text { \% respecto al total } \\
\text { de no nativos }\end{array}$ & Total & $\begin{array}{c}\text { \% respecto al total } \\
\text { de no nativos }\end{array}$ \\
\hline Bulgaria & 20.132 & 13,4 & 24.352 & 12,7 \\
Rumanía & 14.062 & 9,4 & 26.288 & 13,7 \\
Marruecos & 13.209 & 8,8 & 19.840 & 10,3 \\
Portugal & 12.277 & 8,2 & 12.699 & 6,6 \\
Colombia & 10.813 & 7,2 & 14.040 & 5,2 \\
Francia & 9.301 & 6,2 & 8.883 & 4,6 \\
Ecuador & 8.799 & 5,9 & 9.598 & 5,0 \\
Brasil & 5.430 & 3,6 & 5.358 & 2,8 \\
Argentina & 5.223 & 3,5 & 4.698 & 2,4 \\
República Dominicana & 5.108 & 3,4 & 9.828 & 5,1 \\
Total 10 naciones & 104.358 & 69,6 & 131.584 & 68,4 \\
\hline
\end{tabular}

Fuente: Padrones Municipales, 2007 y 2014.

Los datos se refieren a población "nacida en el extranjero", y no a población “extranjera”, para que el análisis no esté distorsionado por el significativo número de personas que han adquirido en estos años la nacionalidad española, fundamentalmente inmigrantes de origen latinoamericano. Esta opción tiene el inconveniente menor — por su escaso número- de estar incluyendo a los hijos de españoles nacidos en el extranjero, y de estar infravalorando la repercusión demográfica de la inmigración extranjera al no tener en cuenta a los hijos de inmigrantes, nacidos ya en España.

Utilizaremos dos fuentes de información: el Padrón Municipal de Habitantes y el Censo de Población de 2011. El Padrón Municipal de Habitantes, a pesar de presentar, como todas las fuentes estadísticas disponibles, problemas para hacer un cómputo certero del número de inmigrantes residentes en nuestro país, sigue siendo la fuente canónica para medir la intensidad de los flujos migratorios (Reher, Requena y Sanz, 2011:25). Hay que tener en cuenta, en cualquier caso, la posible duplicidad de registros, dada la renovación bianual 
del empadronamiento y la elevada movilidad de la población inmigrante, o la repercusión que el endurecimiento de las políticas en relación con el empadronamiento de personas en situación irregular y la restricción de sus derechos haya tenido en el no registro de algunas personas inmigrantes. Utilizaremos esta fuente para analizar las pautas de asentamiento territorial y algunas características demográficas relevantes de la población objeto de estudio. La clasificación de municipios utilizada en los datos padronales se basa en una tipología de municipios elaborada desde el Departamento de Geografía de la Universidad de Valladolid, que permite discriminar los municipios rurales no solo por su tamaño poblacional sino por su dinámica económica y su funcionalidad espacial (Alario y Baraja, 2004). Distinguiremos entre municipios urbanos, que incluyen tanto a los municipios mayores de 10.000 habitantes como aquéllos que, siendo de menor tamaño, se integran en áreas periurbanas o en áreas de influencia urbana, pequeñas ciudades, incluyendo los municipios de 5.000 a 10.000 habitantes, los pueblos grandes — de 2.000 a 5.000 habitantes-y los pueblos pequeños, de hasta 2.000 habitantes.

Del Censo de Población de 2011 obtendremos información sobre las pautas de convivencia familiar y de empleo de los inmigrantes residentes en municipios rurales de Castilla y León, entendiendo en este caso por rurales todos aquéllos con menos de 2.000 habitantes, ya que esta fuente no nos permite discriminar los pequeños municipios que son periurbanos o se encuentran en áreas de influencia urbana.

\section{INMIGRANTES EN EL MEDIO RURAL: ORÍGENES NACIONALES Y PAUTAS DE ASENTAMIENTO TERRITORIAL}

En 2007 la población de origen extranjero en Castilla y León era de casi 150.000 personas, un 5,9\% de la población total, porcentaje bastante inferior a la media nacional $(11,6 \%)$. Los principales grupos según origen geográfico eran los procedentes de la Unión Europea (45\% del total de la población de origen extranjero, de los cuales algo más de la mitad eran rumanos y búlgaros), de América (un 35\% del total, la gran mayoría procedentes de Latinoamérica, siendo los colombianos y ecuatorianos los grupos nacionales más numerosos, un $37 \%$ de todos los latinoamericanos), y de África (un 12\% del total, de los cuales el $73 \%$ eran marroquíes). 
Si atendemos a las pautas territoriales de asentamiento de la población inmigrante en ese momento es innegable que la inmigración extranjera es un fenómeno predominantemente urbano: en 2007 un $68 \%$ viven en municipios urbanos, periurbanos o situados en un área de influencia urbana. La tercera parte restante viven en municipios no urbanos, y aunque su presencia relativa en los municipios rurales más pequeños es muy baja $(4,1 \%)$, en los centros comarcales intermedios es superior a la de los municipios urbanos. Esta pauta de asentamiento se mantendrá básicamente igual a lo largo del periodo considerado.

En los años que transcurren entre 2007 y 2014, el porcentaje de población inmigrante en la región aumenta, y lo hace especialmente en los entornos no urbanos, no solo porque en los primeros cinco años que siguen al estallido de la crisis económica la población de origen extranjero sigue llegando a la región, sino por la dinámica demográfica claramente recesiva de la población autóctona. El resultado de estos dos procesos es que la presencia de población inmigrante es especialmente relevante en las pequeñas ciudades y los pueblos grandes. En conjunto y en términos relativos los municipios no urbanos incrementan su población inmigrante más que los urbanos.

TABLA 2

PORCENTAJE DE POBLACIÓN INMIGRANTES EN CASTILLA Y LEÓN, POR TIPO DE MUNICIPIO, EN 2007 Y 2014

\begin{tabular}{|l|r|r|r|c|}
\hline Tipo de municipios & $\begin{array}{c}\text { Nacidos en } \\
\text { España } \\
\text { (2007) }\end{array}$ & $\begin{array}{c}\text { Nacidos en } \\
\text { el extranjero } \\
\text { (2007) }\end{array}$ & $\begin{array}{c}\text { \% nacidos en } \\
\text { el extranjero sobre } \\
\text { población total } \\
\text { (2007) }\end{array}$ & $\begin{array}{c}\text { \% nacidos en } \\
\text { el extranjero sobre } \\
\text { población total } \\
\text { (2014) }\end{array}$ \\
\hline Urbanos & 63,2 & 68,0 & $6,3 \%$ & $8,1 \%$ \\
Pequeñas ciudades & 5,9 & 7,4 & $7,3 \%$ & $9,5 \%$ \\
Pueblos grandes & 6,2 & 8,1 & $7,5 \%$ & $10,0 \%$ \\
Pueblos pequeños & 24,6 & 16,5 & $4,1 \%$ & $5,5 \%$ \\
\hline TOTAL & $100 \%$ & $100 \%$ & $5,9 \%$ & $7,7 \%$ \\
\hline Total (N) & 2.378 .419 & 149.998 & 2.528 .417 & 2.494 .970 \\
\hline
\end{tabular}

Fuente: Padrón Municipal 2007 y 2014. 
Los datos muestran pautas de asentamiento territorial muy diversas. Frente al asentamiento casi totalmente urbano de los inmigrantes colombianos y ecuatorianos en la comunidad, los inmigrantes procedentes del este de Europa son con mucho los más "rurales": más de la mitad de los búlgaros viven en municipios no urbanos (casi un 30\% en pueblos pequeños) y casi un $45 \%$ de los rumanos están en la misma situación (con uno de cada cinco residiendo en un pueblo pequeño). La población marroquí tiene también una presencia significativa en los entornos rurales, cerca de un $40 \%$ de la misma residen en municipios no urbanos.

\section{GRÁFICO 1}

DISTRIBUCIÓN TERRITORIAL DE LOS CINCO GRUPOS NACIONALES DE POBLACIÓN INMIGRANTE EN CASTILLA Y LEÓN. 2007

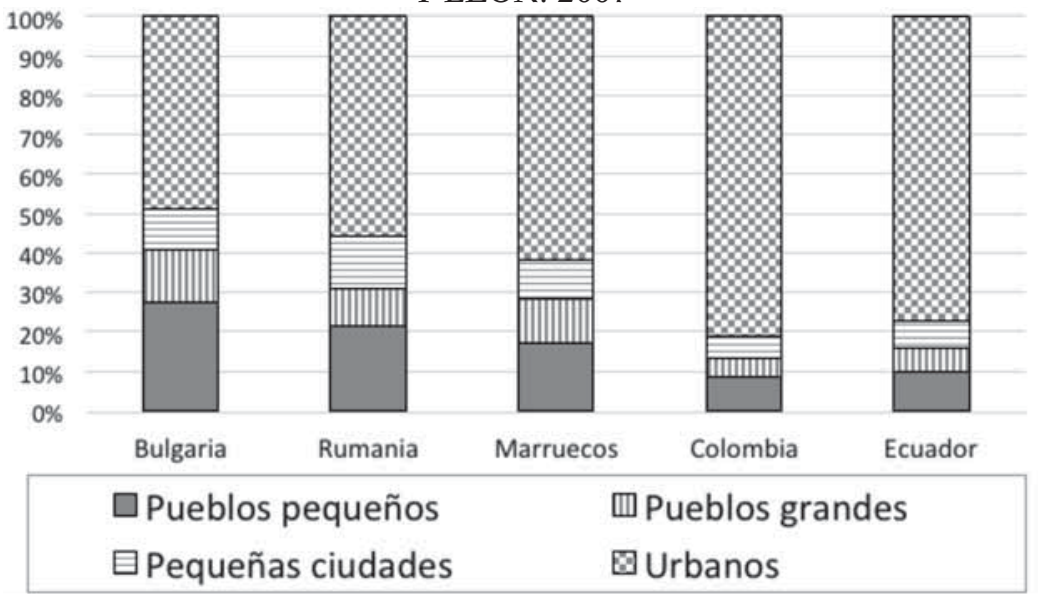

Fuente: Padrón Municipal 2007 y 2014.

Es difícil de encontrar una sola causa a tan diferentes pautas de asentamiento. Hay que tener en cuenta sin duda el funcionamiento de las redes migratorias, la mayor o menor antigüedad de los flujos migratorios, la mayor o menor especialización de determinados grupos nacionales en determinados sectores económicos y en concreto 
en las actividades agrarias, o el origen más o menos rural de los migrantes. Sin embargo, en este caso podemos comprobar cómo la conocida vinculación de los inmigrantes marroquíes a la agricultura no parece corresponderse con una pauta de residencia claramente rural, lo que viene a confirmar que la actividad agraria no se vincula necesariamente a una fuerza de trabajo local y asentada (Camarero, Sampedro y Oliva, 2013). En cualquier caso, la residencia predominantemente urbana de los inmigrantes latinoamericanos en la comunidad sugiere una implicación en las actividades agrarias mucho menor a la que se ha reportado en las comunidades autónomas del levante español, muy vinculada a la una agricultura intensiva orientada a la exportación que sin duda no tiene una relevancia comparable en Castilla y León (Castellanos y Pedreño, 2001; Pedreño y Riquelme, 2006).

Esta pauta de asentamiento, que hace de los inmigrantes búlgaros, rumanos y marroquíes los más presentes en las áreas rurales, permanece prácticamente inalterada en los años de la crisis económica, salvo una cierta reducción de la presencia de los inmigrantes del este de Europa en los pueblos más pequeños que se produce a partir de 2012 y que analizaremos más adelante.

\section{ESTRATEGIAS FAMILIARES Y ARRAIGO RURAL: FAMILIAS NACIONALES, FAMILIAS TRANSNACIONALES Y FAMILIAS INTERNACIONALES}

La heterogeneidad de la inmigración de origen extranjero no acaba en las pautas de asentamiento territorial sino que se extiende a una composición por sexo de las poblaciones extremadamente diferente y que ya ha sido referenciada en otros estudios relativos a las estrategias familiares de migración (Camarero y García, 2004; Camarero 2010). La población marroquí es una población que presenta una elevada masculinización; los inmigrantes latinoamericanos, por el contrario, son en su mayor parte mujeres. Los inmigrantes búlgaros y rumanos presentan una composición por sexo mucho más equilibrada, siendo con todo todavía predominante la presencia masculina. Este peculiar rasgo demográfico es un primer indicador de la existencia de estrategias migratorias muy diferentes. 
TABLA 3

RAZÓN ENTRE LOS SEXOS (MUJERES POR 100 HOMBRES)

EN LOS CINCO GRUPOS NACIONALES DE POBLACIÓN INMIGRANTE, POR TIPO DE MUNICIPIO, EN 2007

\begin{tabular}{|l|c|c|c|c|c|}
\hline \multicolumn{1}{|c|}{ Tipo de municipios } & Bulgaria & Rumania & Marruecos & Colombia & Ecuador \\
\hline Urbanos & 91 & 96 & 55 & 153 & 109 \\
Pequeñas ciudades & 87 & 84 & 64 & 157 & 90 \\
Pueblos grandes & 89 & 81 & 50 & 153 & 112 \\
Pueblos pequeños & 83 & 83 & 48 & 150 & 107 \\
\hline TOTAL & 88 & 90 & 54 & 153 & 108 \\
\hline
\end{tabular}

Fuente: Padrón Municipal 2007.

Es reseñable el hecho de que en los grupos con mayor presencia en entornos rurales la razón entre los sexos tienda a ser más desequilibrada en ellos, lo que podría indicar la mayor facilidad para la reagrupación familiar en entornos urbanos y en general en los núcleos rurales con mayor tamaño.

La amplia literatura académica sobre las migraciones que existe ya en nuestro país evidencia la existencia de diferentes estrategias familiares de inmigración, estrategias que favorecen más o menos la movilidad individual o familiar, femenina o masculina, de personas con o sin pareja o hijos en el país de origen. De igual forma determinados modelos facilitan más o menos la reagrupación familiar y/o la dilatan más o menos en el tiempo. También existen muy diferentes pautas en relación a la propensión a establecer matrimonios endogámicos — dentro de la propia comunidad étnica o nacional- o fuera de ella, con otros inmigrantes o con la población autóctona de la sociedad de acogida.

Sabemos así que la población marroquí se caracteriza por ser una migración fundamentalmente masculina, con un considerable número de matrimonios y familias transnacionales, produciéndose la reagrupación familiar de esposas e hijos cuando la situación del pionero masculinoestáconsolidada.Lainmigraciónlatinoamericana, aunque con diversidades internas, se distingue por la frecuente migración de mujeres, en muchas ocasiones cabezas de familia monomarentales que tras un tiempo reagrupan a sus hijos y forman 
nuevas parejas en España, muchas veces con hombres españoles. Los inmigrantes procedentes del Este de Europa presentan una pauta de migración diferente: hombres y mujeres migran por igual, la reagrupación familiar se produce de una manera relativamente rápida, y se observa un alto nivel de matrimonios endogámicos, es decir, con compatriotas. Estas diferentes estrategias tienen no solo su reflejo en la razón entre los sexos existente en las distintas poblaciones sino, como vemos a continuación, en los modelos de convivencia familiar en el medio rural de Castilla y León.

\section{TABLA 4}

FORMAS DE CONVIVENCIA DE LOS INMIGRANTES DE 30 A 50 AÑOS, EN LOS CINCO GRUPOS NACIONALES, EN LOS MUNICIPIOS DE MENOS DE 2.000 HABITANTES

\begin{tabular}{|c|c|c|c|c|c|c|c|c|c|c|}
\hline \multirow{3}{*}{ Formas de convivencia } & \multicolumn{10}{|c|}{ Población } \\
\hline & \multicolumn{2}{|c|}{ búlgara } & \multicolumn{2}{|c|}{ rumana } & \multicolumn{2}{|c|}{ marroquí } & \multicolumn{2}{|c|}{ colombiana } & \multicolumn{2}{|c|}{ ecuatoriana } \\
\hline & $H$ & $M$ & $H$ & $M$ & $H$ & $M$ & $H$ & $M$ & $H$ & $M$ \\
\hline En pareja & 84,7 & 80,9 & 71,5 & 68,3 & 59,2 & 68,0 & 59,6 & 41,7 & 72,3 & 54,7 \\
\hline En pareja con español/a & 1,0 & 4,6 & 0,9 & 8,5 & 2,9 & 9,3 & 6,5 & 40,8 & 8,2 & 39,2 \\
\hline Monoparental & 2,3 & 2,5 & 1,6 & 10,6 & 3,6 & 8,1 & 1,7 & 9,3 & 8,3 & 3,2 \\
\hline Con sus padres & 3,0 & 0,7 & 0,4 & 0,2 & 1,4 & 0,0 & 1,1 & 1,6 & 0,3 & 0,0 \\
\hline Solo/a o en hogar sin núcleo & 9,0 & 11,3 & 25,6 & 12,4 & 32,9 & 14,6 & 31,1 & 6,6 & 10,8 & 3,0 \\
\hline TOTAL & $100 \%$ & $100 \%$ & $100 \%$ & $100 \%$ & $100 \%$ & $100 \%$ & $100 \%$ & $100 \%$ & $100 \%$ & $100 \%$ \\
\hline Total en convivencia familiar & 91 & 88,7 & 74,4 & 87,6 & 67,1 & 85,4 & 68,7 & 93,4 & 89,1 & 97,1 \\
\hline
\end{tabular}

Fuente: Censo de Población, 2011.

En la Tabla 4 se recogen dichos modelos de convivencia, referidos a los individuos de entre 30 y 50 años, grupo de edad en el que los proyectos y biografías familiares suelen estar ya completadas. Se han distinguido diferentes formas de convivencia familiar, entendiendo por ésta aquella que existe cuando hay en el hogar un núcleo basado en las relaciones de pareja o en las relaciones paternofiliales. Podemos encontrarnos así a inmigrantes que viven en pareja —con o sin hijos-; sin pareja pero con sus hijos —en hogares monoparentales-, o con sus padres. Hemos diferenciado también, 
dentro de las parejas, aquéllas en las que uno de los cónyuges es español, para calibrar la importancia de las uniones con personas de la sociedad de acogida. Hay que tener en cuenta, en todo caso, que al clasificar a los sujetos por país de nacimiento, podríamos estar considerando matrimonios mixtos a aquellos en que uno de los cónyuges ha nacido en España a pesar de ser de origen extranjero.

Los datos muestran que existe una nítida diferencia entre hombres y mujeres: las mujeres presentan de forma casi general unas formas de convivencia más familiar. Salvo en el caso de la población búlgara, en que las diferencias son mínimas, los hombres viven más frecuentemente solos o con personas con las que no forman un núcleo familiar. La causa es doble: por un lado el carácter masculino de muchas corrientes migratorias hace que los hombres actúen como pioneros, llegando las mujeres posteriormente como reagrupadas, y por tanto, ya con una familia. Por otro lado, como algunos estudios han mostrado, las mujeres inmigrantes tienen una posición muy diferente a la de los varones en el mercado matrimonial: ellas se ven favorecidas por el mayor número de parejas potenciales en su propio grupo étnico o nacional, pero también les resulta mucho más fácil emparejarse fuera de ese grupo (Sánchez Domínguez, de Valk y Reher, 2011).

La segunda constatación es que las diferentes estrategias migratorias de los grupos nacionales se reflejan claramente en las formas de convivencia familiar. El modelo marroquí, con un alto porcentaje de convivencia no familiar masculina, nos remite a la estrategia de emigración masculina y el mantenimiento de familias transnacionales que pueden dejar de serlo al cabo de cierto tiempo, con la reagrupación de la esposa que reside en Marruecos. El modelo de los países de Europa del Este se refleja en una mayor similitud en las formas de convivencia de hombres y mujeres búlgaros y rumanos, con la salvedad ya reseñada de una mayor frecuencia, aunque no excesiva, de matrimonios mixtos entre las mujeres. Estas pautas reflejan un modelo de migración mucho más familiar en que los inmigrantes casados lo hacen con su pareja e hijos o los reagrupan rápidamente, y en la que los solteros se casan ya en España con compatriotas, algo que se ve favorecido por un flujo migratorio que se ha producido de una manera muy rápida y con gran intensidad (Sánchez Domínguez, de Valk y Reher, 2011).

La población latinoamericana se caracteriza por el elevado porcentaje de matrimonios mixtos de las mujeres: en torno a un $40 \%$ de las mujeres colombianas y ecuatorianas residentes en el medio 
rural están casadas con hombres nacidos en España. Las poblaciones colombiana y ecuatoriana se distinguen por las diferentes pautas de convivencia familiar que presentan los hombres: mientras los colombianos tienen una pautas más cercanas a las de los marroquíes — pocos casados y muchos viviendo en hogares sin núcleo-, los ecuatorianos se asemejan más a los migrantes de Europa del Este. Esto puede reflejar el carácter heterogéneo de los flujos migratorios ecuatorianos, parte de los cuales responden a una migración de tipo familiar, y parte a una migración fundamentalmente femenina (Camarero y García, 2004).

Si contrastamos los datos referidos a la estructura de la convivencia familiar de los inmigrantes en los municipios rurales con la de los municipios mayores de 10.000 habitantes podemos observar una tendencia clara a formas de convivencia más familiares en el medio urbano que en el medio rural.

\section{TABLA 5}

PORCENTAJE DE INMIGRANTES DE 30 A 50 AÑOS, QUE VIVEN EN HOGARES FAMILIARES, SEGÚN LOS CINCO GRUPOS NACIONALES, SEXO Y MUNICIPIO DE RESIDENCIA

\begin{tabular}{|c|c|c|c|c|c|c|c|c|c|c|}
\hline \multirow{3}{*}{$\begin{array}{l}\text { Total en convivencia } \\
\quad \text { familiar: }\end{array}$} & \multicolumn{10}{|c|}{ Población: } \\
\hline & \multicolumn{2}{|c|}{ búlgara } & \multicolumn{2}{|c|}{ rumana } & \multicolumn{2}{|c|}{ marroquí } & \multicolumn{2}{|c|}{ colombiana } & \multicolumn{2}{|c|}{ ecuatoriana } \\
\hline & $H$ & $M$ & $H$ & $M$ & $H$ & $M$ & $H$ & $M$ & $H$ & $M$ \\
\hline Municipios > 10.000 hab. & 91,5 & 93,7 & 81,7 & 94,2 & 73,1 & 95,4 & 81,8 & 88,3 & 74,9 & 94,4 \\
\hline Municipios $<2.000$ hab. & 91,0 & 88,7 & 74,4 & 87,6 & 67,1 & 85,4 & 68,7 & 93,4 & 89,1 & 97,1 \\
\hline
\end{tabular}

Fuente: Censo de Población, 2011.

Este hecho sugiere que, al menos para los inmigrantes económicos, el proceso de consolidación familiar es más fácil en entornos urbanos. Esto abundaría en la condición de "estación de paso" de los entornos rurales, en los que se mantiene la residencia únicamente hasta conseguir mejorar la propia situación legal y/o económica, accediendo a mercados de trabajo más atractivos, lo que permite proceder a la reagrupación de la familia o la formación de 
una nueva en el país de acogida. Los grupos nacionales en los que más claramente se observa esta pauta son los búlgaros, rumanos y marroquíes, precisamente aquellos que tienen una mayor presencia en el medio rural.

En el caso de los inmigrantes de origen latinoamericano, observamos que mientras los hombres colombianos aumentan el carácter familiar de su convivencia en los municipios urbanos, los ecuatorianos se vinculan a los entornos rurales de forma familiar, y al urbano de forma no familiar. Este último hecho puede estar relacionado con la ya señalada heterogeneidad de la migración ecuatoriana, que incluye muy variados y diferentes perfiles familiares - tanto mujeres cabezas de familia que emigran solas, como varones de la misma condición, como solteros de ambos sexos - y laborales con dos perfiles binómicos: uno mayoritario de residentes en núcleos urbanos vinculados al servicio doméstico, la hostelería y la construcción; y otro minoritario, vinculado al medio rural y al trabajo agrario (Camarero y García, 2004).

$\mathrm{Si}$ las estrategias matrimoniales de inmigrantes colombianas y ecuatorianas se caracterizan por la importancia del matrimonio con españoles, esto se acentúa en los municipios rurales: el porcentaje de matrimonios mixtos es $40,8 \%$ y $39,3 \%$ respectivamente en los municipios rurales, frente a un 23,9\% y 13,9\% respectivamente en los municipios urbanos. El elevado nivel de masculinización y de soltería masculina en las áreas rurales de Castilla y León parece un elemento claro que favorece la formación de ese tipo de familias. Esta pauta distintiva de la inmigración latinoamericana ha sido ampliamente documentada a nivel general (Sánchez Domínguez, de Valk y Reher, 2011) y tiene evidentemente una repercusión en las áreas rurales. La significativa presencia de mujeres inmigrantes en las denominadas "caravanas de mujeres" — surgidas por los elevados niveles de soltería masculina en los pueblos- puede ser un indicador en este sentido (Bodoque, 2010).

Es reseñable que las únicas excepciones respecto a la pauta de una mayor convivencia familiar de las mujeres en el medio urbano es la de ecuatorianas y colombianas: para ellas la posibilidad de consolidación familiar está más vinculada al medio rural, vía matrimonio con españoles. En definitiva la población inmigrante latinoamericana, la menos presente en el medio rural de la comunidad, se vincula a él fundamentalmente a través del matrimonio con autóctonos y a través de formas familiares de convivencia. 
Podríamos así resumir señalando que los entornos urbanos parecen seguir siendo territorio más proclive a las formas familiares de convivencia, y que los cinco grupos nacionales analizados ponen en práctica estrategias migratorias en las que cumplen un papel muy relevante las familias nacionales -en el caso de los migrantes búlgaros y rumanos-, las familias transnacionales —en el caso de los marroquíes - y las familias internacionales —en el caso de las mujeres latinoamericanas-.

\section{PAUTAS DE EMPLEO DE LOS INMIGRANTES EN EL MEDIO RURAL: ORÍGENES NACIONALES Y ESPECIALIZACIÓN SECTORIAL Y POR GÉNERO}

El análisis de las pautas de empleo de la población inmigrante residente en el medio rural reviste una considerable dificultad debido a la falta de representatividad que las principales fuentes estadísticas laborales presentan en relación con el hábitat rural, y la inexistencia de este tipo de información en las estadísticas padronales. La Encuesta Nacional de Inmigración (ENI) de 2007 confirmó lo que numerosos estudios sobre el terreno habían ya apuntado: los mercados de trabajo donde se integran los trabajadores inmigrantes llegados a las áreas rurales se concentran en un abanico reducido de actividades y ocupaciones, que están a su vez muy diferenciadas por sexo. Los hombres aparecen empleados fundamentalmente en la construcción $(32,6 \%)$, las manufacturas $(20,5 \%)$ y la agricultura $(12 \%)$ y las mujeres en el comercio (17\%), la hostelería $(18,4 \%)$ y el servicio doméstico (16,2\%) (Camarero, Sampedro y Oliva, 2013). Por otro lado el análisis de la evolución de las trayectorias laborales apunta, igualmente, a que el abandono del trabajo agrario parece constituir un paso adelante: para los varones la agricultura es el sector que se constituye como primera ocupación al llegar a España; para las mujeres el servicio doméstico. Estos sectores van siendo abandonados por el empleo en la construcción y en la manufactura, en el caso de los primeros, y por la hostelería y el comercio para las segundas. Hay que tener en cuenta que el trabajo agrario y la residencia rural no son dos condiciones necesariamente unidas. Por paradójico que pueda parecer, la ocupación agraria no implica arraigo rural. Al menos a nivel nacional, los datos de la ENI 2007 
muestran cómo ni siquiera la cuarta parte de los trabajadores agrarios reside en municipios rurales, mientras que por el contrario, la tercera parte de quienes trabajan en la agricultura residen en municipios mayores de 50.000 habitantes (Camarero, Sampedro y Oliva, 2013). La movilidad espacial juega en este caso un papel muy relevante, en la medida en que residencia rural y trabajo urbano representan una pauta utilizada tanto por la población autóctona como inmigrante.

La ENI de 2007 mostraba también la existencia de una fuerte vinculación entre la actividad agraria y ciertas nacionalidades: marroquíes, ecuatorianos y rumanos agrupaban a más del $50 \%$ de los extranjeros ocupados en este sector, aunque ni siquiera para estos colectivos la agricultura representaba su principal fuente de empleo. La especialización de determinadas nacionalidades en determinados empleos depende en parte de un fenómeno que se ha denominado etnoestratificación del mercado de trabajo, y que se refiere a la forma en que los empleadores apelan a diferencias culturales para legitimar una clasificación de las aptitudes y cualificaciones laborales por nacionalidad (Pedreño, 2005; Pedreño y Riquelme, 2006).

\section{TABLA 6}

EMPLEO DE LOS INMIGRANTES RESIDENTES EN MUNICIPIOS MENORES DE 2000 HABITANTES, POR SECTOR DE ACTIVIDAD, ORIGEN NACIONAL Y SEXO

\begin{tabular}{|l|rr|rr|rr|rr|rr|}
\hline \multicolumn{1}{|c}{ Sector de actividad } & \multicolumn{10}{|c|}{ Población: } \\
\cline { 2 - 12 } & \multicolumn{2}{|c|}{ búlgara } & \multicolumn{1}{c|}{ rumana } & marroquí & colombiana & ecuatoriana \\
\cline { 2 - 11 } & $\boldsymbol{H}$ & $\boldsymbol{M}$ & $\boldsymbol{H}$ & $\boldsymbol{M}$ & $\boldsymbol{H}$ & $\boldsymbol{M}$ & $\boldsymbol{H}$ & $\boldsymbol{M}$ & $\boldsymbol{H}$ & $\boldsymbol{M}$ \\
\hline Agricultura & 45 & 30 & 47 & 31 & 41 & 4 & 23 & 8 & 17 & 3 \\
Construcción & 17 & 2 & 20 & 1 & 33 & 3 & 18 & - & 35 & 3 \\
Industria, Transporte, Energía & 19 & 9 & 17 & 11 & 10 & 7 & 13 & 7 & 19 & 6 \\
Comercio, hostelería & 12 & 29 & 9 & 33 & 8 & 40 & 17 & 37 & 19 & 28 \\
Servicio Doméstico & - & 14 & - & 7 & - & 20 & 2 & 20 & - & 28 \\
Salud y servicios sociales & 1 & 6 & - & 5 &. & 14 & 6 & 12 & 1 & 19 \\
Otros servicios & 6 & 10 & 7 & 12 & 8 & 12 & 21 & 16 & 9 & 13 \\
\hline TOTAL & $100 \%$ & $100 \%$ & $100 \%$ & $100 \%$ & $100 \%$ & $100 \%$ & $100 \%$ & $100 \%$ & $100 \%$ & $100 \%$ \\
\hline
\end{tabular}

Fuente: Censo de Población, 2011. 
Si analizamos las pautas de empleo que presentan en 2011 los inmigrantes que residen en el medio rural de Castilla y León lo primero que llama la atención en estos datos, respecto a los proporcionados a nivel nacional por la ENI 2007, es la pérdida de importancia del empleo en la construcción para los varones, como efecto del impacto de la crisis en este sector. Solo para los inmigrantes ecuatorianos la construcción sigue representando su principal sector de empleo. La agricultura y los sectores industriales absorben ahora el empleo masculino de los residentes rurales.

Hay dos diferencias muy relevantes entre los cinco grupos considerados: en primer lugar la implicación mucho más intensa de la población marroquí, búlgara y rumana en la agricultura frente a una implicación mucho más modesta de colombianos y ecuatorianos. En segundo lugar, la existencia en la población marroquí, colombiana y ecuatoriana de una clara división del trabajo según el género, de forma tal que el empleo masculino en agricultura y construcción contrasta claramente con un empleo femenino que se concentra en el sector servicios: comercio y hostelería en primer lugar; servicio doméstico en segundo, y servicios relacionados con la salud y la asistencia social en tercer lugar. Por el contrario, las pautas de empleo de las poblaciones del Este de Europa se caracterizan por una alta implicación de hombres y mujeres en la agricultura, con casi una tercera parte de las mujeres implicadas en actividades agrarias, casi el mismo número que las empleadas en comercio y hostelería. Esta alta implicación de hombres y mujeres en las actividades agrarias sugiere la existencia de un tipo de empleo agrario realizado en grupos familiares frente a modelos más individualizados de inserción laboral.

\section{CRISIS Y EVOLUCIÓN DE LA PRESENCIA DE INMIGRANTES EN EL MEDIO RURAL: DEL ÉXODO QUE NUNCA SE PRODUJO A LOS EFECTOS VISIBLES DE LA CRISIS}

Entre 2007 y 2012 los datos padronales muestran una evolución similar de la población inmigrante en la mayoría de las comunidades autónomas: se frena el enorme crecimiento de la población inmigrante, pero su volumen no disminuye, manteniéndose ligeros 
incrementos anuales. El desplome de la construcción y la repercusión de la crisis económica en el resto de los sectores que emplean mayoritariamente a los migrantes, no parece por tanto provocar procesos de retorno masivo, a pesar de la persistencia de elevados niveles de desempleo tanto en la población inmigrante como en la población autóctona a lo largo de esos cinco años.

En Castilla y León, al igual que sucede en el ámbito nacional, no se produce una reducción de la población de origen extranjero. Más bien al contrario esta población crece entre 2007 y 2012, contribuyendo de una forma clara al mantenimiento demográfico de la comunidad: en este periodo la región pierde casi 42.000 nativos pero gana algo más de 59.000 nacidos en el extranjero, pasando ésta a representar un 8,2 por ciento de la población total.

En los gráficos siguientes podemos comparar la evolución de la población inmigrante en los municipios urbanos y en los pequeños pueblos Como podemos comprobar el incremento de la población inmigrante es similar en entornos rurales y urbanos, y especialmente significativo en el caso de los rumanos y los marroquíes. Una diferencia fundamental entre ambos colectivos es que mientras en el caso de la población rumana la razón entre los sexos no varía en el periodo considerado (pasando de 90 mujeres por 100 hombres a 89 mujeres por 100 hombres), en el caso de la población marroquí este indicador varía de forma muy significativa hacia un mayor equilibrio entre los sexos: de 54 mujeres por cada 100 hombres en 2007 a 67 mujeres por cada 100 hombres en 2012. Este "reequilibrio" demográfico es, si cabe, aún más importante en los pequeños pueblos donde la relación pasa de 48 a 60 mujeres por cada 100 hombres. En un contexto de aumento de la población inmigrante ello solo puede deberse a procesos de reagrupación familiar. Por el contrario, el incremento de la población rumana se produce sin que, aparentemente, la crisis haya tenido una repercusión reseñable en la composición de los flujos demográficos. 
GRÁFICOS 2 Y 3

EVOLUCIÓN RELATIVA DE LOS CINCO GRUPOS NACIONALES

ENTRE 2007 Y 2012, SEGÚN TIPO DE MUNICIPIO (2007=100)
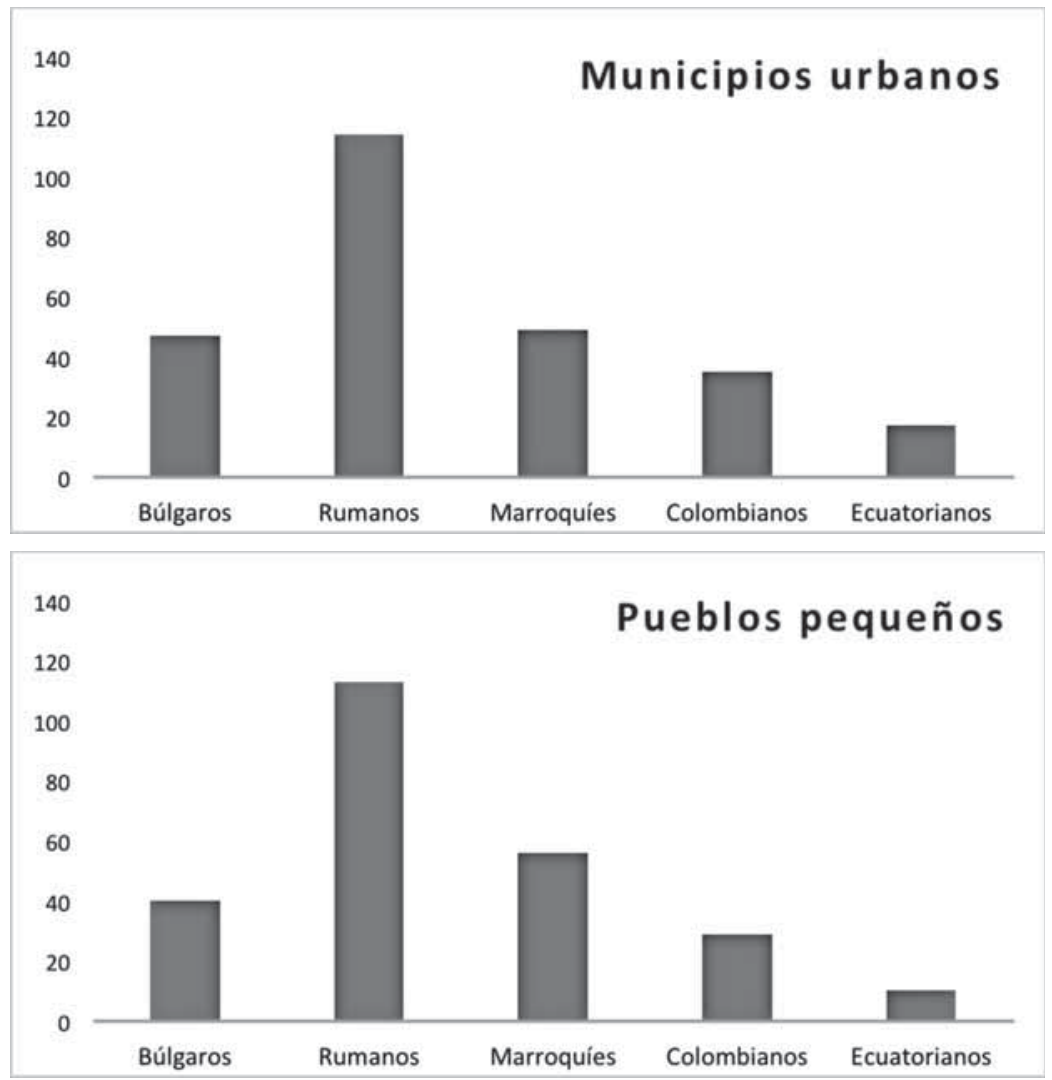

Fuente: Padrón Municipal 2007 y 2012.

Entre 2012 y 2014 los registros padronales ya dejan ver una reducción en números absolutos de la población de origen extranjero a nivel nacional y en todas las comunidades autónomas.

Si volvemos a la evolución de las cifras de los cinco grupos nacionales que estamos considerando, entre 2012 y 2014 las mayores reducciones porcentuales se producen entre la población búlgara, que pierde cerca de 5.000 efectivos (un 17\% del total) y rumana, con una pérdida de algo más de 3.600 personas (un 12\% del total). 
La población marroquí permanece prácticamente invariable y los grupos latinoamericanos experimentan pérdidas mucho menores, de un 3\% la población colombiana, y de un 7\% la población ecuatoriana. En los dos gráficos siguientes vemos reflejada la dimensión territorial de estas pérdidas poblacionales: la reducción es mucho mayor en los pueblos más pequeños que en los municipios urbanos, para todos los grupos nacionales, salvo para la población marroquí que aumenta su presencia en estos núcleos.

\section{GRÁFICOS 4 y 5}

EVOLUCIÓN RELATIVA DE LOS CINCO GRUPOS NACIONALES ENTRE 2012 Y 2014, SEGÚN TIPO DE MUNICIPIO (2012=100)

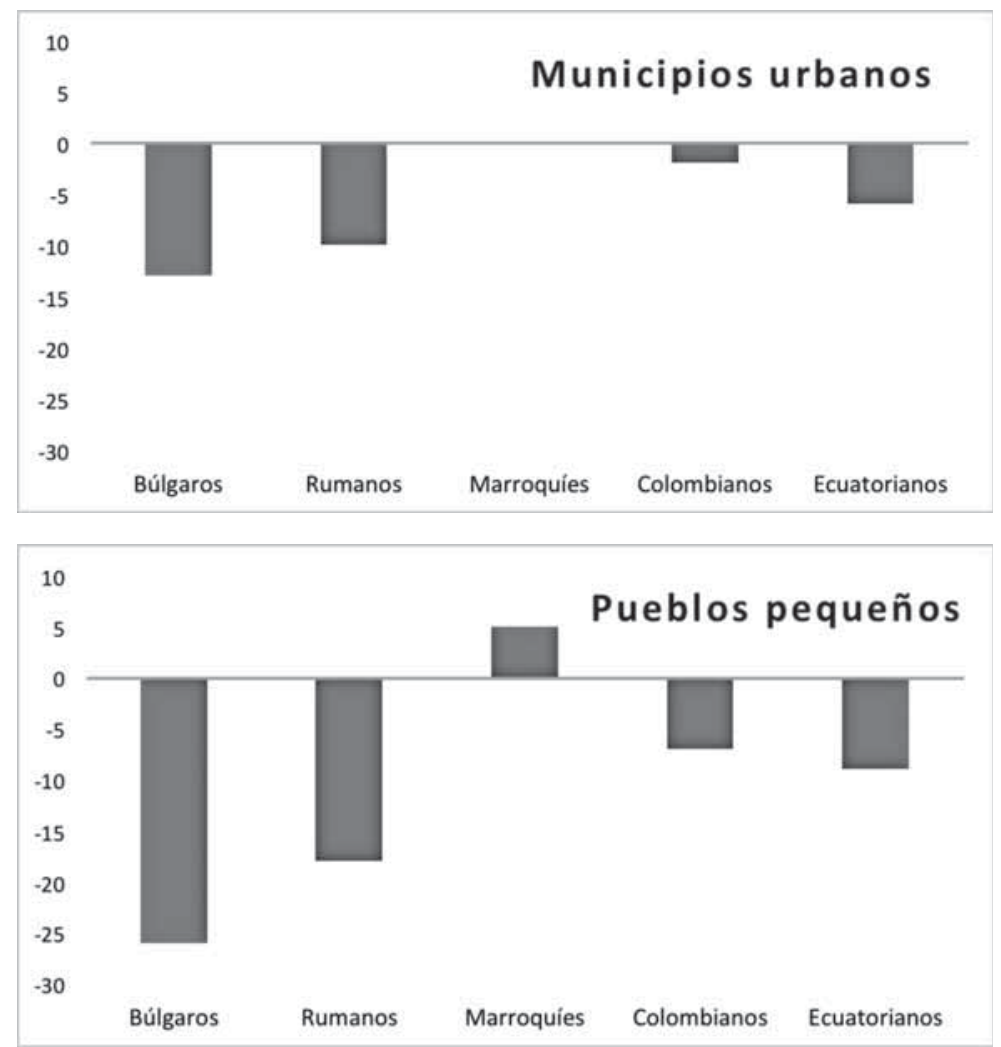

Fuente: Padrón Municipal 2012 y 2014. 
La evolución de la razón entre los sexos nos da, de nuevo, información adicional sobre la composición de los flujos migratorios en la comunidad. En todos los grupos que ven disminuir sus efectivos aumenta la presencia relativa de mujeres, lo que indica que la crisis expulsa fundamentalmente a varones, por la mayor incidencia de la misma en los sectores de empleo masculino - fundamentalmente la construcción- pero también, probablemente, por el mayor arraigo familiar de las mujeres. En el caso del colectivo marroquí su composición por sexo también evoluciona hacia una mayor presencia femenina (de 67 a 71 mujeres por cada 100 hombres; en el caso de los pequeños pueblos, de 60 a 65 mujeres por cada 100 hombres), lo que indica, en un contexto de mantenimiento o ligero incremento de la población, una continuación de procesos de reagrupación familiar. Estudios realizados con algunos de estos mismos colectivos en las comunidades del arco mediterráneo apuntan, al igual que aquí, a un proceso de retorno minoritario y selectivo - jóvenes varones fundamentalmente-, y descubren la complejidad de los movimientos transnacionales que provoca la crisis (Torres y Gadea, 2010). Estos incluirían tanto el retorno al país de origen, propiamente dicho, como la migración a terceros países, las migraciones circulares (combinación de periodos en España y periodos en los países de origen) o procesos de transnacionalización familiar a partir de la migración o retorno de una parte de los miembros de la unidad familiar (Torres, 2014).

En definitiva se aprecia un impacto muy diferente de la crisis en los distintos colectivos: los grupos procedentes de Europa del Este, experimentan una evolución muy irregular con crecimientos muy significativos en los primeros años de la crisis económica y una caída igualmente brusca en los dos últimos años del periodo considerado. Por el contrario la población marroquí ha ido consolidando su presencia en Castilla y León, y en concreto en el medio rural, experimentando una significativa evolución en su composición por sexo hacia un mayor equilibrio, lo que puede con bastante seguridad relacionarse con procesos de reagrupación familiar.

Si consideramos las distintas estrategias familiares y pautas de empleo de los diferentes grupos nacionales, los datos apuntan a que existen algunas que resultan más resilientes ante la crisis y que por tanto se vinculan al arraigo y la integración de la población inmigrante en el medio rural. 


\section{CONCLUSIÓN Y DISCUSIÓN}

La capacidad de los núcleos rurales para retener a medio y largo plazo a una población inmigrante que es estratégica para su sostenibilidad demográfica y social depende tanto de las características de dichos núcleos como de las que presentan estos nuevos residentes. Frente a argumentos frecuentemente manejados en los discursos políticos y mediáticos, como la "cercanía cultural" - un concepto especialmente difuso y engañoso-, o la concepción de la población inmigrante como un mero recurso laboral cuya permanencia es función directa de su implicación en los trabajos que la población autóctona no está en posición o disposición de realizar, consideramos que hay otros elementos que pueden ser importantes tanto para aumentar su capacidad de hacer frente a los efectos de la crisis como para favorecer su arraigo en el medio rural. La forma en que los inmigrantes vinculan sus proyectos migratorios con la formación de sus hogares y familias, y las pautas de empleo sobre las que se organiza la supervivencia individual y familiar son dos de esos elementos.

El análisis realizado en estas páginas nos ha permitido comprobar la existencia de muy diferentes modelos de asentamiento territorial, estrategias familiares de migración y pautas de empleo en diferentes grupos nacionales. Aquí, al igual que en muchos otros estudios sobre migraciones, se parte de la consideración de los inmigrantes como actores sociales, que en tanto que tales despliegan una diversidad de estrategias para mejorar su situación en el marco de sus posibilidades, considerando que la unidad de análisis de dichas estrategias no es el individuo, sino la unidad familiar (Torres, 2014: 217).

De la atenta lectura a los datos se deduce que ciertas estrategias familiares de migración parecen favorecer la permanencia de los inmigrantes en las áreas rurales, en concreto aquéllas que posponen la reagrupación de familias transnacionales hasta que la posición del pionero masculino se ha consolidado, propia de la población marroquí, y aquéllas que se vinculan al matrimonio de mujeres inmigrantes con hombres españoles, presente especialmente en las poblaciones de origen latinoamericano, en nuestro caso, colombiana y ecuatoriana. Estas dos estrategias familiares aparecen vinculadas a unas pautas de empleo claramente especializadas por género, en las que las mujeres están prácticamente ausentes de la agricultura, dedicándose mayoritariamente a actividades terciarias. 
Por el contrario, las pautas presentes en las poblaciones procedentes del Este de Europa, con modelos de migración altamente familiares y una elevada implicación de hombres y mujeres en las actividades agrarias parecen ser definitivamente más vulnerables frente a los efectos de la crisis, ya que han sido inmigrantes búlgaros y rumanos los que más directamente han desaparecido del medio rural y de la región cuando los efectos de la crisis se han dejado notar con mayor intensidad a partir de 2012.

Este análisis nos conduce a plantearnos la relación que existe entre estrategia familiar migratoria, como composición de formas de hogar y de actividad, y temporalidad de la estancia. Si bien por sentido común parecería que formas migratorias más familiares se asociarían a posiciones a la larga de mayor arraigo, los datos han mostrado que esto no es precisamente así. La intensa llegada de población del Este de Europa a las áreas rurales, población que viene en grupos nucleares y familiares y que desarrolla también economías familiares, se corresponde con un mayor abandono de la residencia rural. Son emigraciones que podemos considerar nomádicas, familias que varían su residencia en función de las coyunturas económicas. Son además comparativamente poco resilientes, por la baja especialización tanto interna que desarrollan sus miembros como externa en la diversificación de modelos de actividad.

Por el contrario, las migraciones que se desarrollan con modelos progresivos de asentamiento familiar, con familias transnacionales que consideran la reagrupación tras una consolidación previa de la posición del pionero, han resultado los modelos que producen mayor arraigo. Son estructuras más resilientes en la medida en que pueden, según la coyuntura, adelantar o posponer el reagrupamiento dentro de una estrategia de expectativas a largo plazo, y por ello a larga ser más estables.

Es difícil valorar el efecto que la crisis tendrá sobre el arraigo definitivo de esta población en áreas rurales, pero lo que sí que es cierto que ha supuesto un golpe importante al equilibrio demográfico y a la sostenibilidad social, auténtica espada de Damocles del hábitat rural.

Indudablemente es necesaria investigación cualitativa que nos permita entender los efectos de la crisis en las estrategias y trayectorias laborales y familiares, así como el conjunto de elementos que intervienen en la integración de la población inmigrante, no siendo los menores la percepción que de la inmigración tiene la población 
autóctona y los principales actores políticos e institucionales en el ámbito local. Por el momento, los datos estadísticos nos confirman en la absoluta necesidad de adoptar una perspectiva familiar cuando analizamos los comportamientos migratorios.

\section{REFERENCIAS BIBLIOGRÁFICAS}

Alario, M. y Baraja, E. (2004): “Características y tipificación de los espacios rurales” en M. Alario (dir.), Las mujeres en el medio rural de Castilla y León. Valladolid, Consejo Económico y Social de Castilla y León, pp. 49-66.

Arango, J. (2012): "Del boom a la crisis: la inmigración en España en la primera década del siglo XXI" en Matia Portilla, F. J. (dir.), Crisis e Inmigración. Reflexiones interdisciplinares sobre la Inmigración en España. Valencia, Tirant Lo Blanch, pp. 23-55.

- (1999): "Becoming a Country of Immigration at the end of the XXth Century: the Case of Spain" en KInG, R., Lazaridis, G. y Tsardanitiss, C. (eds): Eldorado or Fortress? Migration in Southern Europe. London, Palgrave McMillan, pp. 253-276.

BAYONA, J. y GIL, F. (2013): "Is foreign immigration the solution to rural depopulation?” Sociologia Ruralis, 53(1), pp. 26-51.

Bodoque, Y. (2010): “Caravanas de mujeres: etnografía de una modalidad de encuentro amoroso”. Ankulegi, 14, pp. 93-103.

CAmarero, L. (2013): Tiempos de crisis en las áreas rurales. Las sociedades adaptativas. XI Congreso Español de Sociología. Madrid. 10-12 Julio, 2013.

- (2010): “Transnacionalidad Familiar: Estructuras familiares y trayectorias de reagrupación de los inmigrantes en España”. Empiria, 17, pp. 39-71.

Camarero, L. (coord.); Cruz, F.; González, M.; del Pino, J. A.; Oliva, J. y SAMPEDRO, R. (2009): La población rural en España. De los desequilibrios a la sostenibilidad social. Barcelona, Fundación La Caixa, Colección de Estudios Sociales, $\mathrm{n}^{\circ} 27$.

Camarero, L. y García, I. (2004): “Los paisajes familiares de la inmigración”. Revista Española de Sociología, 4, pp. 173-198.

Camarero, L.; Sampedro, R. y Oliva, J. (2013): “Trayectorias ocupacionales y residenciales de los inmigrantes extranjeros en las áreas rurales españolas". Sociología del Trabajo, n 77, pp. 69-91.

- (2011): "Foreigners, Neighbours, Immigrants: Translocal Mobilities in Rural Areas in Spain” en Hedberg, CH. y Carmo, R.M. (eds), Translocal Ruralism. Mobility and Connectivity in European Rural Spaces. Heidelberg-New York, Springer, 2011, pp. 143-162. 
Castellanos, M. L y Pedreño, A. (2001): “Desde El Ejido al accidente de Lorca. Las amargas cosechas de los trabajadores inmigrantes en los milagrosos vergeles de la agricultura mediterránea”. Sociología del Trabajo, n 42 , pp. 3-32.

Consejo Económico y Social de Castilla y León (2012): Población y poblamiento en Castilla y León. Informe a Iniciativa Propia IIP1/12. Valladolid, Consejo Económico y Social de Castilla y León.

Collantes, F.; Pinilla, V.; Sáez, L. A. y Silvestre, J. (2014): "Reducing Depopulation in Rural Spain: the Impact of Immigration". Population, Space and Place, 20 (7), pp. 606-621.

EsPARcia, J. (2002): "La creciente importancia de la inmigración en las zonas rurales de la comunidad valenciana”. Cuadernos de Geografía, 72, pp. 289-306.

Fonseca, M. L. (2008): "New Waves of Immigration to Small Towns and Rural Areas in Portugal”. Population, Space and Place, 14, pp. 525-535.

García Coll, A. y SÁnchez, D. (2005): “La población rural en Cataluña: entre el declive y la revitalización”. Cuadernos Geográficos, 36 (1), pp. 387-407.

Hoggart, K y Mendoza, C. (1999): “African Immigrant Workers in Spanish Agriculture”. Sociologia Ruralis, 39 (4), pp. 538-562.

IBÁÑEZ ANgulo, M. (2006): Inserción Laboral e integración social de la población inmigrante en Castilla y León. Valladolid, Junta de Castilla y León, Consejería de Familia e Igualdad de Oportunidades.

Jentsch, B. (2009): "Migrant Integration in Rural Areas. Evidence from New Countries of Immigration". International Journal of Multicultural Studies, 9 (1), pp. 1-12.

Kasimis, C. (2008): "Survival and expansion. Migrants in Greek Rural Regions”. Population, Space and Place, 14, pp. 511-524.

Kasimis, Ch.; Papadoulos, A. G. y Pappas, C. (2010): "Gaining from Rural Migrants: Migrant Employment Strategies and Socioeconomic Implications for Rural Labour Markets”. Sociologia Ruralis, 50 (3), pp. 258-276.

King, R. (2000): "Southern Europe in the changing global map of migration" en King, R.; LAzaridis, G. y Tsardanidis, C. (eds), Eldorado or fortress? Migration in southern Europe. London, Palgrave Macmillan, pp.3-26.

Mancilla, C.; Viladomiou, L. y Guallarte, C. (2010): “Emprendimiento, inmigrantes y municipios rurales: el caso de España”. Economía Agraria y Recursos Naturales, 10 (2), pp. 121-142.

MendozA, C. (2003): Labour Immigration in Southern Europe. African Employment in Iberian Labour Markets. Aldershot, Ashgate

Miguélez, F. (dir.); Martín, A.; Alós-Moner, R.; Esteban, F.; López, P.; Molina, O. y Moreno, S. (2011): Trayectorias Laborales de los inmigrantes en España. Barcelona. Barcelona, Fundación La Caixa.

Moral, A. del y Martín, A. (2014): "Efectos de la crisis sobre los flujos migratorios: un análisis provincial” en Matia Portilla, F. J. (dir.), Nuevos retos para la integración social de los inmigrantes. Valencia, Tirant Lo Blanch, pp. 359-386. 
MuÑoz, M. (coord.) (2005): Trabajadores inmigrantes en la construcción y en la agricultura. Toledo, Comisiones Obreras Castilla-La Mancha.

Oriz, D.; Arnalte, E. y Moraguess, A. M. (2013): "What role for southern European agriculture in the period of crisis? Some hypothesis and preliminary results from Spain". XXV European Congress of Rural Sociology. Firenze (Italia) 29 July- 1 August, 2013.

Pedone, C. (2005): "Diversificación de las cadenas migratorias ecuatorianas hacia el trabajo agrícola de Murcia, España” en Pedreño, A. y Hernández, M. (coords.), La condición inmigrante. Murcia, Universidad de Murcia, pp. 255-271.

Pedreño, A. (1999): “Construyendo la Huerta de Europa: Trabajadores Sin Ciudadanía y Nómadas Permanentes en la Agricultura Murciana”. Migraciones, $\mathrm{n}^{\circ}$ 5, pp. 87-120.

- (2005): "Sociedades etnofragmentadas" en Pedreño, A. y Hernández, M. (coords.), La condición inmigrante. Murcia, Universidad de Murcia, pp. 75-103.

Pedreño, A. y Riquelme, P. (2006): "La condición inmigrante de los trabajadores rurales". Revista Española de Estudios Agrosociales y Pesqueros, 211, pp. 189-238.

Pinilla, V., Ayuda, M. I. y Sáez, L. A. (2008): "Rural Depopulation and the Migration Turnaround in Mediterranean Westhern Europe: A Case Study in Aragon”. Journal of Rural and Community Development, 3, pp. 1-22.

Prieto, S. y Papadodima, Z. (2010): "Reversión (comparativa) del despoblamiento rural a través de las migraciones internacionales”. AGER. Documento de Trabajo $\mathrm{n}^{\circ}$ 28- 2010.

Pumares, P.; García A. y Asensio, A. (2006): La movilidad laboral y geográfica de la población inmigrante. Madrid, Ministerio de Trabajo y Asuntos Sociales.

Reher, D.; Requena, M. y SAnz, A. (2011): “¿España en la encrucijada? Consideraciones sobre el cambio de ciclo migratorio". Revista Internacional de Sociología, Monográfico no 1 Abril-2011, pp. 9-44.

Reher, D. y Silvestre, J. (2011): “Internal migration patterns of foreingborn immigrants in Spain. A study based on the National Immigrant Survey (ENI-2007)". Revista Internacional de Sociología, Monográfico $\mathrm{n}^{\circ}$ 1 Abril-2011, pp. 167-188.

Requena, M., y SÁnchez-Dominguéz, M. (2011): "Las familias inmigrantes en España”. Revista Internacional de Sociología, Monográfico $\mathrm{n}^{\circ} 1$ Abril-2011, pp. 79-104.

Roquer, S. y Blay, J. (2008): “Del éxodo rural a la inmigración extranjera: el papel de la población extranjera en la recuperación demográfica de las zonas rurales españolas (1996-2006)”. Scripta Nova, XII-270 (129).

SÁnchez-Domínguez, M.; de VALK, H. y Reher, D. (2011): “Marriage strategies among immigrants in Spain”. Revista Internacional de Sociología, Monográfico no 1 Abril-2011, pp. 139-166. 
Sánchez-Flores, S.; Royo, I.; Lacomba, J.; Mari, E. y Benlloch, C. (2014): "Mujeres inmigrantes emprendedoras en el medio rural. Factor para la sostenibilidad económica y social de las áreas rurales en la Comunidad Valenciana”. AGER, n 16 , pp. 69-109.

SAmpedro, R. (2012): "La paradoja de la "condición inmigrante": una reflexión desde la sociología sobre la integración social de los inmigrantes” en MATIA Portilla, F. J. (dir.), Crisis e Inmigración. Reflexiones interdisciplinares sobre la Inmigración en España. Valencia, Tirant Lo Blanch, pp. 55-89.

Soronellas, M.; Bodoque, Y; Blay, J: Roquer, S. y Torrens, R. (2014): "Inmigrar a la Cataluña rural. Contextos de ruralidad e itinerarios migratorios de mujeres extranjeras hacia pequeños municipios”. AGER, $\mathrm{n}^{\mathrm{o}} 16$, pp. 111-148.

SolÉ, A. (2010): “Características sociodemográficas, pautas de distribución territorial y proceso migratorio de la población de nacionalidad extranjera en el Alt Pirineu i Aran: contribuciones a la transformación de un espacio de montaña”. AGER. Documento de trabajo n 29-2010.

Torres Pérez, F. (2014): “Crisis y estrategias de los inmigrantes en España: el acento latino”. Revista CIDOB d'Afers Internacionals, n ${ }^{\circ} 106-107, \mathrm{pp}$. 215-236.

Torres Pérez, F. y Gadea, E. (2010): “Inserción laboral de los inmigrantes, estructura etno-fragmentada y crisis económica. El caso del Campo de Cartagena (Murcia)”. Sociología del Trabajo, nº 69, pp. 61-81. 